\title{
Novel Operation Mode for Eliminating Influence of Inclination Angle and Friction in Atomic Force Microscopy
}

\author{
Fei WANG, Yueyu WANG, Faquan ZHOU and Xuezeng ZHAO
}

School of Mechanical and Electronic Engineering, Harbin Institute of Technology, Harbin, Heilongjiang 150001, P. R. China.

It is well know that, in constant-force mode of contact atomic force microscopy (AFM), the cantilever deflection can be used as input to a feedback circuit that moves the scanner up and down in $z$, responding to the topography by keeping the cantilever deflection constant. In this case, with the cantilever deflection held constant, the total force applied to the sample, which refers to van der Waals $(\mathrm{vdW})$ force in most situations, is constant. As a result, the tip-sample distance remains constant. One can deduce that the accuracy in topography imaging depends on the one-to-one corresponding relationship between the cantilever deflection and the tip-sample distance; whereas such relationship can not be always achieved in practice in the presence of the friction and the inclination angle of sample surface ${ }^{[1-3]}$. See Fig.1, when the tip is scanning along the sample surface, the interactive force between the tip and the sample has two contributors, $F_{n}$ and $F_{f}$, where $F_{n}$ is the vdW force and $F_{f}$ is the frictional force. $F_{z}$ and $F_{x}$ are the component force of $F_{\text {todal }}$ in the $x o z$ coordinates and it is easily known that $F_{z}=F_{n} \cos (\theta) \pm \mu F_{n} \sin (\theta)$ and $F_{x}=-F_{n} \sin (\theta) \pm \mu F_{n} \cos (\theta)$, where $\theta$ and $\mu$ refers to the inclination angle and the friction coefficient. Importantly, one can conclude here that $F_{z}$, which is linearly related to the cantilever deflection by its spring constant, is influenced by $\theta$ and $\mu$ effectively besides the vdW force, which breaks the one-to-one corresponding relationship between the cantilever deflection and the tip-sample distance.

Recently, we developed a novel operation mode, in which the effect of inclination angle and friction on the contact mode of AFM has been eliminated. In the new mode, AFM tip keep contacting with the sample surface, the scanning direction is vertical with the long axis of cantilever (lateral), see Fig.2. We obtain $F_{z}$ and $F_{x}$ by multiplying the AFM signals and the corresponding sensitivity. Then the vdW force $F_{n}$ can be obtained from $F_{n}^{2}+\left(\mu F_{n}\right)^{2}=F_{z}^{2}+F_{x}^{2}$. Consequently, $d$ can be obtained from its relationship with the vdW force $F_{n} . \theta$ can be obtained from the government equation for $F_{z}$ and $F_{x}$ from $F_{n}, \mu$ and $\theta$ stated in the last paragraph. At the last, the topography can be obtained as $Q_{z}=t_{z}-S_{z}-d|\cos (\theta)|$ and $Q_{x}=s_{x}-t_{x}+d|\sin (\theta)|$, where $t_{z}$ and $t_{x}$ refer to tip deflections at the sampling point, which can be obtained through $t_{z}=F_{z} / k_{z}$ and $t_{x}=F_{x} / k_{x}$, where $k_{z}$ and $k_{x}$ refer to the normal and lateral spring constant of the cantilever. $s_{z}$ and $s_{x}$ refer to scanner displacements. $Q_{z}$ and $Q_{x}$ are the ultimately position of the sampling point in the zox coordinate. During the measurement process, we control the AFM feedback to make the scanner move the sample along $\mathrm{Z}$-axis and keep the vdW force $F_{n}$ as constant as well as records the $Q_{z}$ and $Q_{x}$ at every sampling points, consequently, the reconstructed of sample surface topography height is obtained.

We have improved our AFM to enable the new operation mode for validation. Two sets of comparative experiments have been done: 1) Imaging a sampling line along a flat silicon surface under both the constant force contact mode and the new mode we presented to check the effect of friction force on their images, see Fig.3; 2) Imaging a sampling line with slopes under both the ordinary and our modes to check the effect of inclination angle on their images, see Fig.4. The results show a good agreement with our description in the last paragraph that the effect of frictional force 
and inclination angle on topography imaging in contact mode of AFM can be eliminated or at least deceased effectively by working under the new operation mode we presented.

\section{Reference}

[1] S. A. Edwards, W.A. Ducker and J.E. Sader. J. Appl. Phys, 103(6), 064513 (2008).

[2] G. Shang, X. Qiu, C. Wang and C. Bai. Appl. Phys. A: Mater. Sci. Proc., 66: S333-S335, (1998)

[3] J. P. Aime, Z. Elkaakour, S. Gauthier, et al. Surface Science, 329(1): 149-156(1995)

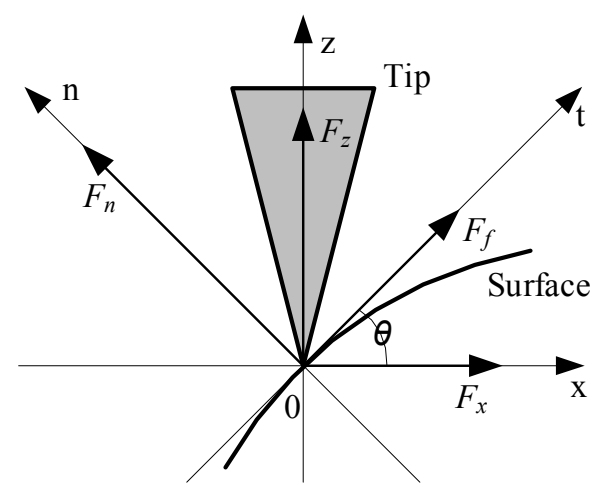

Fig.1 Force applied on the tip in contact mode in the new operation mode.

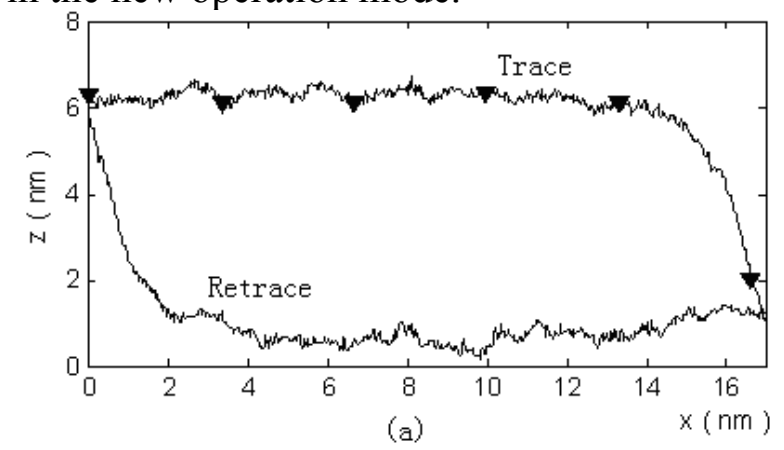

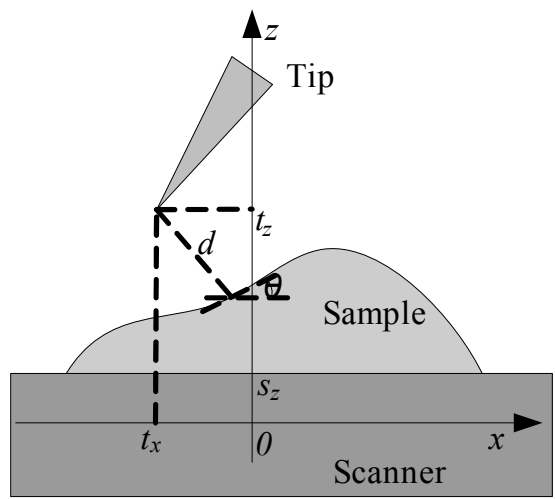

Fig.2 Diagram for surface topography

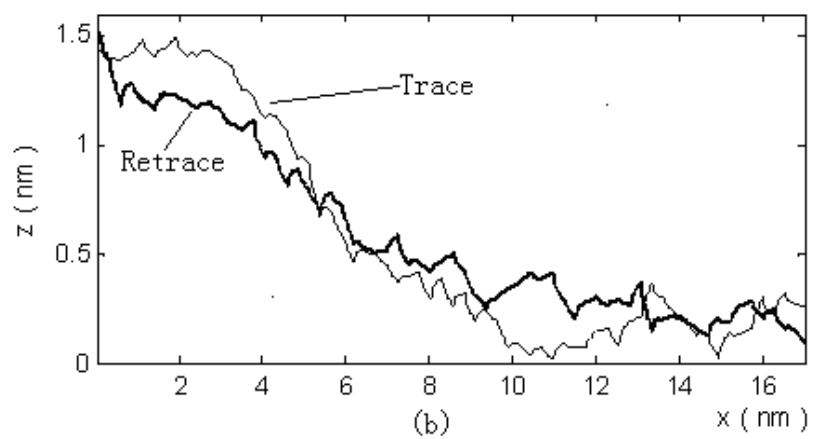

Fig. 3 Topography of a line on a flat silicon surface under different working mode: (a) ordinary force-constant contact mode; (b) the new operation mode presented.
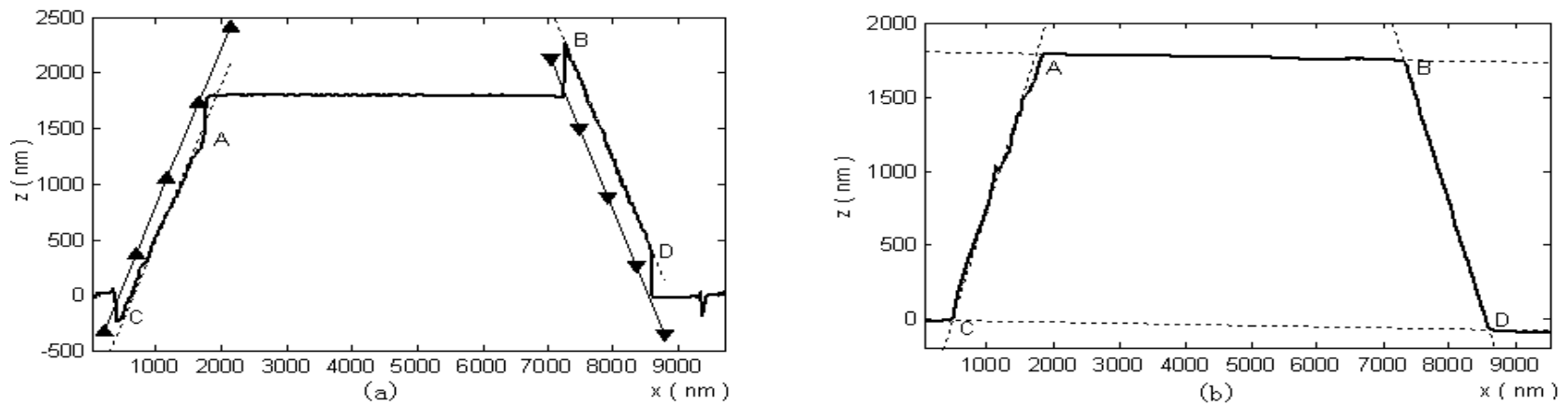

Fig. 4 Topography of a scanning on a surface of TGF11 from Mikromasch under different working mode: (a) ordinary force-constant contact mode; (b) the new operation mode we presented. 